\title{
Alternative interval estimation for parameters of bivariate exponential model with time varying covariate
}

\begin{abstract}
This paper investigates several alternative methods of constructing confidence interval estimates based on the bootstrap and jackknife techniques for the parameters of a parallel two-component system model with dependent failure and a time varying covariate, when data is censored. This model is an extension of the bivariate exponential model. Bootstrap confidence interval techniques, the bootstrap-t, bootstrap-percentile andBCamethods are compared with the confidence interval based on the jackknife via coverage probability study using simulated data. The results clearly indicate that the jackknife technique works far better than any of the bootstrap techniques when dealing with censored data.
\end{abstract}

Keyword: Bootstrap, Jackknife, Censoring, Covariates 